\title{
Effect of Gibberellic Acid and Harvesting Time on the Seed Quality of Four Okra Cultivars
}

\author{
Ghadir Mohammadi ${ }^{1}$, Ebrahim M. Khah ${ }^{1}$, Spyridon Alexandros Petropoulos ${ }^{1}$, Dimosthenis B. Chachalis ${ }^{2}$, Fariba \\ Akbari $^{3} \&$ Garip Yarsi ${ }^{4}$ \\ ${ }^{1}$ Department of Agriculture, Crop Production and Rural Environment, School of Agricultural Sciences, \\ University of Thessaly, VOLOS, Greece \\ ${ }^{2}$ Benaki Phytopathological Institute, ATHENS, Greece \\ ${ }^{3}$ Jihad-Agriculture Education Center of Sarpol-e-Zahab, Kermanshah, Iran \\ ${ }^{4}$ Plant and Animal Production Department, Vocational School of Silifke, University of Mersin, MERSIN, \\ Turkey \\ Correspondence: Spyridon Alexandros Petropoulos, Department of Agriculture, Crop Production and Rural \\ Environment, School of Agricultural Sciences, University of Thessaly, VOLOS, Greece. E-mail: \\ fangio57gr@gmail.com
}

Received: February 19, 2014 Accepted: March 21, 2014 Online Published: June 15, 2014

doi:10.5539/jas.v6n7p200 URL: http://dx.doi.org/10.5539/jas.v6n7p200

\begin{abstract}
In an experiment at the University of Thessaly during the summer season of 2011, the effect of foliar application of gibberellic acid $\left(\mathrm{GA}_{3}\right)$ to okra at an early stage of plant growth (3-4 leaves) on plant growth, pod and seed characteristics was studied in relation to harvest time. $\mathrm{GA}_{3}$ was applied at concentrations of 0 (Control), 50, and $100 \mathrm{mg} \mathrm{L}^{-1}$ to four okra cultivars ('Boyiatiou', 'Veloudo', 'Clemson' and 'Pylaias') and pods were harvested 30, 35, 40 and 50 days after anthesis (DAA) from the lower part of the plant. From the results it was found that $\mathrm{GA}_{3}$ application increased plant height irrespective of cultivar and $\mathrm{GA}_{3}$ concentration $\left(50\right.$ and $\left.100 \mathrm{mg} \mathrm{L}^{-1}\right)$, but without increasing flower induction or pod set. Similarly, $\mathrm{GA}_{3}$ had no effect on pod dimensions (which were determined by genotype) or mean 100 seed weight, except in Boyiatiou. Similarly, $\mathrm{GA}_{3}$ application did not consistently affect seed moisture content, but it did however, increase the number of seeds per pod. Germination was either promoted ('Veloudo'), inhibited ('Boyiatiou') or not affected ('Pylaias', 'Clemson') by $\mathrm{GA}_{3}$. Differences in germination were apparently related to the incidence of hard seeds. Storage of seeds for 18 months improved germination. Overall, pod and seed characteristics were affected more by genotype and harvest time than by $\mathrm{GA}_{3}$ application.
\end{abstract}

Keywords: gibberellic acid; okra; seed germination; seed moisture; seed yield; seed hardness

Abbreviations: $\mathrm{GA}_{3}$ - gibberellic acid, DAA - days after anthesis, DAT - days after transplantation

\section{Introduction}

Gibberellic acid $\left(\mathrm{GA}_{3}\right)$ or gibberellins comprise a group of naturally occurring plant hormones which play a central role in the early germination processes of seeds by activating enzyme production and mobilizing storage reserves (Bewley \& Black, 1983). Additionally, foliar application of gibberellins stimulates and synchronizes flowering and fruit set (Briant, 1974), as well as enhancing photosynthesis and growth (Yuan \& Xu, 2001), or stimulating growth but not the rate of photosynthesis (Dijkstra \& Kuiper, 1989).

Okra seeds frequently exhibit seed hardness and physical dormancy, which complicates crop management because of non-uniform germination after sowing resulting in a lack of crop uniformity and causing difficulties in weed control, harvest time etc. (Mohammadi, Khah, \& Bannayan, 2011). Several publications have reported that soaking okra seeds in growth regulators, particularly gibberellin, increases germination (Nandpuri, Sooch, \& Randhawa, 1969) possibly due to a reduction in seed hardness (Passam \& Polyzou, 1997). On the other hand, Pal and Hossain (2001) found no effect of $\mathrm{GA}_{3}$ on okra seed germination and seed weight, but a significant effect of this hormone on plant height and the number of pods per plant.

The positive effect of $\mathrm{GA}_{3}$ on plant height has been widely reported for many crops, including okra (Ilias, Ouzounidou, Giannakoula, \& Papadopoulou, 2007). Moreover, foliar application of $\mathrm{GA}_{3}(20-40 \mathrm{ppm})$ and NAA 
(50-100 ppm) on okra plants was found to increase the number of pods per plant and the number and weight of seeds (Asghar, Hussain \& Ali, 1997; Abduljabbar, Abduljabbar, \& Shukri, 2007). Similarly, Dhankhar and Singh (2009) reported that $\mathrm{GA}_{3}$ application increased okra pod length and diameter. These results could be attributed mainly to the stimulatory effect of $\mathrm{GA}_{3}$ on plant growth, which resulted in higher rates of biosynthesis and therefore higher amounts of assimilates available for distribution to the pods.

The objective of this study was to determine the effect of foliar application of gibberellic acid on the pod and seed quality of four okra genotypes in relation to the time of harvest and the position of the pods on the plant.

\section{Material and Methods}

\subsection{Plant Material and Experimental Conditions}

The experiment was carried out at the experimental farm of the University of Thessaly, Greece, throughout the growing season of 2011. The sand, silt and clay content of the soil at the experimental site was 48,29 and $23 \%$, respectively. The $\mathrm{pH}$ of the soil was 7.7-8.1 and the organic matter content was $1.3 \%$. The soil was a clay loam Inceptisol, typic Xerochrept. Fertilization and crop management were carried out according to standard procedures. Fertilizers were applied as base and side dressing (a total of 30, 35 and $60 \mathrm{Kg} \mathrm{ha}^{-1}$ of N, P and K respectively).

Gibberellic acid $\left(\mathrm{GA}_{3}\right)$ was applied as a foliar spray at three concentrations: $\mathrm{GA}_{3}(1)=0.5 \mathrm{~g} \mathrm{GA}_{3}$ in 1 litre water; $\mathrm{GA}_{3}(2)=1 \mathrm{~g} \mathrm{GA}_{3}$ in 1 litre water and $\mathrm{GA}_{3}(0)=0 \mathrm{~g} \mathrm{GA}_{3}$ in 1 litre water to four cultivars of okra, namely cv. 'Clemson' (very productive with medium sized pods and seeds with high germination percentage), 'Boyatiou' (early and very productive cultivar with small, pentagonal fruits), 'Veloudo' (a very productive cultivan with small, pentagonal fruits and seeds with high germination percentage) and 'Pylaias' (very productive cultivar when irrigated, with small, pentagonal fruits suitable for processing).

The seeds of the four okra cultivars were sown in seed trays containing a peat-based substrate on 20 May and held under controlled temperature $\left(20^{\circ} \mathrm{C}\right)$ until transplantation. Plants were transplanted to the field at the stage of 3-4 true leaves on 10 June. $\mathrm{GA}_{3}$ was dissolved in water $\left(\mathrm{GA}_{3}(1)=0.5 \mathrm{~g} \mathrm{GA}_{3}\right.$ in 1 litre water; $\mathrm{GA}_{3}(2)=1 \mathrm{~g} \mathrm{GA}_{3}$ in 1 litre water and $\mathrm{GA}_{3}(0)=0 \mathrm{~g} \mathrm{GA}_{3}$ ) and applied to the plants twice: (1) at a plant height of approximately $50 \mathrm{~cm}$, (2) 10 days after the first application. All plants were sprayed at the same time of application. The crop was irrigated regularly on a weekly basis with a drip irrigation system, whereas for weed control, Fusillade herbicide (fluazifop-P-butil, $800 \mathrm{ml} / 1000 \mathrm{~m}^{2}$ ) was applied when needed. Anthesis was first recorded 29 days after transplantation (DAT), and from the on each flower was tagged on emergence and its position and the date of flowering were recorded. Harvesting was carried out at four different time periods after anthesis $(30,35,40$ and 45 DAA) for seed quality tests.

\subsection{Measurements}

Seed germination tests were conducted using 100 seeds per treatment, with four replications. The germination tests were carried out on seeds after extraction from the pods either immediately after harvest. Just prior to the germination tests, seeds were surface sterilized first with Chlorine Dioxide, 1 to 20 parts deionised water for $10 \mathrm{sec}$. and then Mancozeb fungicide ( $86 \%$ ) and placed in sterilized Petri dishes on a double layer of Whatman No. 1 filter paper moistened with distilled water. The Petri dishes were placed in the dark in an incubator at $25^{\circ} \mathrm{C}$ for 21 days and every 2 days germinated seeds were counted and removed and, in the case of moisture deficiency, distilled water was added. Seeds were considered germinated when the tip of the radical had grown free of the seed coat (Wiese \& Binning, 1987; Auld et al., 1988). Those seeds which did not germinate and remained rigid after the time period of 21 days in the incubator were considered to be hard seeds, but without testing by scarification (Passam and Polyzou, 1997) or another means, e.g. tetrazolium (Moore, 1985). Germination percentage was calculated by the following formula (ISTA, 2009).

The rate of germination, or germination speed, of the seed lots was derived from the formula:

\section{Mean Germination Time $=\Sigma \mathrm{n} / \Sigma(\mathrm{n}$ X Dn $) \times 100$}

\section{Rate of Germination $=1 /$ mean germination Time}

Where, $\mathrm{n}$ is the number of seeds which germinated on day $\mathrm{n}$. $\mathrm{D}$ is the number of days counted from the beginning of the germination test, $\Sigma$ is the total days of germination period, $\mathrm{R}$ is speed or rate of germination.During cultivation, at each harvest the following parameters were recorded: total flower induction per plant, the number of pods set per plant, pod length and diameter $(\mathrm{cm})$, the average number of seeds per pod, the 100 seeds weight $(\mathrm{g})$ and plant height. Seed germination was tested before and after storage for 18 months at room temperature $\left(25^{\circ} \mathrm{C}\right)$. 


\subsection{Experimental Design and Statistical Analysis}

The experiment was laid out according to a Randomized Complete Block design with factorial arrangement, with each block being replicated three times and consisting of 12 plots of $9 \mathrm{~m}^{2}(3 \times 3 \mathrm{~m})$ each, i.e. a total of 36 plots with 30 plants per plot.

Statistical analysis was carried out with the aid of the S.A.S. statistical package (SAS Institute Inc., USA) and mean comparison was made according to Duncan's Multiple Range Test (DMRT) and the Least Significant Difference (LSD) test at $\mathrm{p} \leq 0.05$. Graphs were generated by using Microsoft Excel software (Microsoft Corporation, USA).

\section{Results}

\subsection{Climatic Conditions}

The climatic conditions of the experimental site are given in Figure 1. The temperature gradually increased from May to July and then decreased till October. There was a difference of about $12-16^{\circ} \mathrm{C}$ between minimum and maximum temperatures (in mean overall differences) throughout the experimental period. There was no rainfall during July, but during the other months varied from 23 to $43 \mathrm{~mm}$; relative humidity thus decreased during July, but afterwards increased progressively. This means that as the number of days from anthesis increased (from 30-50 DAA) pods were increasingly exposed to lower temperatures and higher humidity.

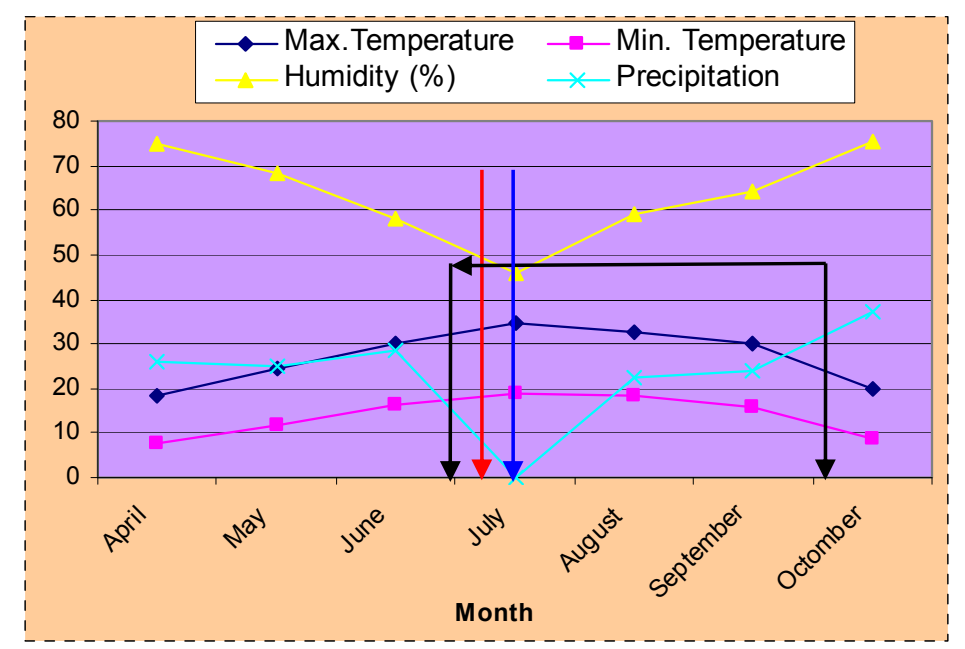

Figure 1. Climatic conditions during the experiment 2011 (black arrows show the duration of pod harvest, the red arrow shows the time of first $\mathrm{GA}_{3}$ application and the blue arrow shows the time of second $\mathrm{GA}_{3}$ application) 


\subsection{Effect of Gibberellic Acid on Flower Induction and Pod Set}

The application of $\mathrm{GA}_{3}$ had no significant effect on flower induction or the number of pods per plant in any of the cultivars (Table 1). Irrespective of $\mathrm{GA}_{3}$ application, both flower induction and pod set were significantly higher in cv. 'Clemson' than in the other three cultivars.

Table 1. The effect of gibberellic acid $\left(\mathrm{GA}_{3}\right)$ on flower induction and pod set

\begin{tabular}{|c|c|c|c|}
\hline Cultivar (C) & $\begin{array}{l}\text { Gibberellic acid rate } \\
\quad\left(\mathrm{mg} \mathrm{L}^{-1}\right)\left(\mathrm{GA}_{3}\right)\end{array}$ & $\begin{array}{l}\text { Total flower induction } \\
\text { (flowers/plant) }\end{array}$ & $\begin{array}{c}\text { Pod set } \\
\text { (pods/plant) }\end{array}$ \\
\hline & $\mathrm{GA}_{3}(1)$ & $13.0^{\mathrm{a}}$ & $11.2^{\mathrm{a}}$ \\
\hline \multirow[t]{2}{*}{ 'Boyiatiou' } & $\mathrm{GA}_{3}(2)$ & $13.2^{\mathrm{a}}$ & $11.4^{\mathrm{a}}$ \\
\hline & $\mathrm{GA}_{3}(0)$ & $11.8^{\mathrm{a}}$ & $9.9^{\mathrm{a}}$ \\
\hline Mean & & 12.6 & 10.8 \\
\hline \multirow[t]{2}{*}{ LSD } & & 2.7 & 2.8 \\
\hline & $\mathrm{GA}_{3}(1)$ & $11.5^{\mathrm{a}}$ & $10.2^{\mathrm{a}}$ \\
\hline \multirow[t]{2}{*}{ 'Veloudo' } & $\mathrm{GA}_{3}(2)$ & $15.5^{\mathrm{a}}$ & $13.8^{\mathrm{a}}$ \\
\hline & $\mathrm{GA}_{3}(0)$ & $14.8^{\mathrm{a}}$ & $13.1^{\mathrm{a}}$ \\
\hline Mean & & 13.9 & 12.3 \\
\hline \multirow[t]{2}{*}{ LSD } & & 4.2 & 4.3 \\
\hline & $\mathrm{GA}_{3}(1)$ & $12.0^{\mathrm{a}}$ & $10.9^{\mathrm{a}}$ \\
\hline \multirow[t]{2}{*}{ ‘Pylaias' } & $\mathrm{GA}_{3}(2)$ & $13.5^{\mathrm{a}}$ & $12.1^{\mathrm{a}}$ \\
\hline & $\mathrm{GA}_{3}(0)$ & $12.1^{\mathrm{a}}$ & $10.6^{\mathrm{a}}$ \\
\hline Mean & & 12.5 & 11.2 \\
\hline \multirow[t]{2}{*}{ LSD } & & 3.9 & 3.8 \\
\hline & $\mathrm{GA}_{3}(1)$ & $20.1^{\mathrm{a}}$ & $19.1^{\mathrm{a}}$ \\
\hline \multirow[t]{2}{*}{ 'Clemson' } & $\mathrm{GA}_{3}(2)$ & $20.0^{\mathrm{a}}$ & $18.5^{\mathrm{a}}$ \\
\hline & $\mathrm{GA}_{3}(0)$ & $18.9^{\mathrm{a}}$ & $17.6^{\mathrm{a}}$ \\
\hline Mean & & 19.6 & 18.4 \\
\hline LSD & & 3.7 & 3.8 \\
\hline \multicolumn{4}{|c|}{$\mathrm{Cx} \mathrm{G}$} \\
\hline \multicolumn{2}{|c|}{$\left(\mathrm{Cx} \mathrm{GA}_{3}(1)\right)$} & $*$ & * \\
\hline \multicolumn{2}{|c|}{$\left({\mathrm{C} x ~ G A_{3}}_{3}(2)\right.$} & $*$ & $*$ \\
\hline \multicolumn{2}{|c|}{$\left(\mathrm{Cx} \mathrm{GA}_{3}(0)\right)$} & $*$ & $*$ \\
\hline
\end{tabular}

Mean values for each cultivar separately in the same column followed by different letters differ significantly at $\mathrm{P}=0.05$.

*: statistically significant $(\mathrm{p}<0.05)$; ns: not significant.

$\mathrm{GA}_{3}(1)=50 \mathrm{mg} \mathrm{L}{ }^{-1}$ Gibberellic acid; $\mathrm{GA}_{3}(2)=100 \mathrm{mg} \mathrm{L}^{-1}$ Gibberellic acid; $\mathrm{GA}_{3}(0)=0 \mathrm{mg} \mathrm{L}^{-1}$ Gibberellic acid.

\subsection{Effect of Gibberellic Acid on Pod Length and Diameter}

Despite occasional differences, $\mathrm{GA}_{3}$ had no significant effect on either pod length or pod diameter, irrespective of the time of harvest (Table 2). Overall, pod length did not differ with harvest time, indicating that growth was maximal by 30 DAA, whereas in all cultivars there was a decrease in pod diameter at 40-50 DAA due to progressive drying of the pods. 'Clemson', which had the highest number of pods per plant (Table 1), also had the smallest pods, in terms of length and diameter (Table 2), perhaps as a result of higher inter-pod competition. 
Table 2. The effect of gibberellic acid $\left(\mathrm{GA}_{3}\right)$ on dry pod dimensions (length and diameter) in relation to harvest time

\begin{tabular}{|c|c|c|c|c|c|c|c|c|c|}
\hline \multirow[b]{2}{*}{ Cultivar (C) } & \multirow[b]{2}{*}{$\begin{array}{l}\text { Gibberellic acid rate } \\
\left(\mathrm{mg} \mathrm{L}^{-1}\right)\left(\mathrm{GA}_{3}\right)\end{array}$} & \multicolumn{4}{|c|}{ Mean pod length $(\mathrm{cm})$} & \multicolumn{4}{|c|}{ Mean pod diameter $(\mathrm{cm})$} \\
\hline & & $\begin{array}{c}30 \\
\text { DAA }\end{array}$ & $\begin{array}{c}35 \\
\text { DAA }\end{array}$ & $\begin{array}{c}40 \\
\text { DAA }\end{array}$ & $\begin{array}{c}50 \\
\text { DAA }\end{array}$ & $\begin{array}{c}30 \\
\text { DAA }\end{array}$ & $\begin{array}{c}35 \\
\text { DAA }\end{array}$ & $\begin{array}{c}40 \\
\text { DAA }\end{array}$ & $\begin{array}{c}50 \\
\text { DAA }\end{array}$ \\
\hline & $\mathrm{GA}_{3}(1)$ & $17.1^{\mathrm{a}}$ & $16.4^{\mathrm{a}}$ & $16.8^{\mathrm{a}}$ & $17.1^{\mathrm{a}}$ & $2.2^{\mathrm{a}}$ & $1.7^{\mathrm{a}}$ & $1.9^{\mathrm{a}}$ & $1.8^{\mathrm{a}}$ \\
\hline \multirow[t]{2}{*}{ 'Boyiatiou' } & $\mathrm{GA}_{3}(2)$ & $15.3^{\mathrm{b}}$ & $15.8^{\mathrm{a}}$ & $18.1^{\mathrm{a}}$ & $15.9^{\mathrm{a}}$ & $2.1^{\mathrm{a}}$ & $2.0^{\mathrm{a}}$ & $1.9^{\mathrm{a}}$ & $1.6^{\mathrm{a}}$ \\
\hline & $\mathrm{GA}_{3}(0)$ & $15.5^{\mathrm{b}}$ & $15.1^{\mathrm{a}}$ & $16.9^{\mathrm{a}}$ & $16.8^{\mathrm{a}}$ & $2.0^{\mathrm{a}}$ & $2.0^{\mathrm{a}}$ & $1.8^{\mathrm{a}}$ & $1.9^{\mathrm{a}}$ \\
\hline Mean & & 15.9 & 15.7 & 17.2 & 16.6 & 2.1 & 1.9. & 1.8 & 1.7 \\
\hline \multirow[t]{2}{*}{ LSD } & & 1.5 & 1.8 & 2.1 & 1.7 & 0.2 & 0.3 & 0.3 & 0.2 \\
\hline & $\mathrm{GA}_{3}(1)$ & $18.1^{\mathrm{b}}$ & $18.2^{\mathrm{a}}$ & $20.3^{\mathrm{a}}$ & $16.6^{\mathrm{a}}$ & $2.0^{\mathrm{a}}$ & $1.7^{\mathrm{a}}$ & $2.1^{\mathrm{a}}$ & $1.6^{\mathrm{a}}$ \\
\hline \multirow[t]{2}{*}{ 'Veloudo' } & $\mathrm{GA}_{3}(2)$ & $19.4^{\mathrm{a}}$ & $17.3^{\mathrm{a}}$ & $22.1^{\mathrm{a}}$ & $17.3^{\mathrm{a}}$ & $1.9^{\mathrm{a}}$ & $1.6^{\mathrm{a}}$ & $2.1^{\mathrm{a}}$ & $1.6^{\mathrm{a}}$ \\
\hline & $\mathrm{GA}_{3}(0)$ & $18.5^{\mathrm{b}}$ & $17.3^{\mathrm{a}}$ & $19.6^{\mathrm{a}}$ & $19.2^{\mathrm{a}}$ & $2.0^{\mathrm{a}}$ & $1.8^{\mathrm{a}}$ & $2.0^{\mathrm{a}}$ & $1.7^{\mathrm{a}}$ \\
\hline Mean & & 18.6 & 17.6 & 20.6 & 17.7 & 1.9 & 1.7 & 2.0 & 1.6 \\
\hline \multirow[t]{2}{*}{ LSD } & & 0.8 & 2.7 & 3.7 & 2.2 & 0.1 & 0.3 & 0.3 & 0.2 \\
\hline & $\mathrm{GA}_{3}(1)$ & $18.0^{\mathrm{a}}$ & $19.6^{\mathrm{b}}$ & $19.5^{\mathrm{a}}$ & $18.1^{\mathrm{ab}}$ & $2.0^{\mathrm{a}}$ & $2.0^{\mathrm{a}}$ & $1.8^{\mathrm{b}}$ & $1.7^{\mathrm{a}}$ \\
\hline \multirow[t]{2}{*}{ 'Pylaias’ } & $\mathrm{GA}_{3}(2)$ & $18.4^{\mathrm{a}}$ & $24.9^{\mathrm{a}}$ & $18.4^{\mathrm{a}}$ & $16.0^{\mathrm{b}}$ & $2.0^{\mathrm{a}}$ & $2.0^{\mathrm{a}}$ & $1.9^{\mathrm{ab}}$ & $1.6^{\mathrm{a}}$ \\
\hline & $\mathrm{GA}_{3}(0)$ & $16.7^{\mathrm{a}}$ & $18.8^{\mathrm{b}}$ & $18.6^{\mathrm{a}}$ & $20.4^{\mathrm{a}}$ & $1.9^{\mathrm{a}}$ & $2.0^{\mathrm{a}}$ & $2.0^{\mathrm{a}}$ & $1.8^{\mathrm{a}}$ \\
\hline Mean & & 17.7 & 21.1 & 18.8 & 18.1 & 1.9 & 2.0 & 1.9 & 1.7 \\
\hline \multirow[t]{2}{*}{ LSD } & & 2.3 & 1.9 & 3.6 & 3.5 & 0.2 & 0.3 & 0.1 & 0.2 \\
\hline & $\mathrm{GA}_{3}(1)$ & $13.9^{\mathrm{a}}$ & $15.0^{\mathrm{a}}$ & $14.2^{\mathrm{c}}$ & $15.9^{\mathrm{a}}$ & $1.4^{\mathrm{a}}$ & $1.5^{\mathrm{a}}$ & $1.2^{\mathrm{a}}$ & $1.3^{\mathrm{a}}$ \\
\hline \multirow[t]{2}{*}{ 'Clemson’ } & $\mathrm{GA}_{3}(2)$ & $14.1^{\mathrm{a}}$ & $14.9^{\mathrm{a}}$ & $15.6^{\mathrm{b}}$ & $15.7^{\mathrm{a}}$ & $1.5^{\mathrm{a}}$ & $1.5^{\mathrm{a}}$ & $1.3^{\mathrm{a}}$ & $1.2^{\mathrm{a}}$ \\
\hline & $\mathrm{GA}_{3}(0)$ & $14.9^{\mathrm{a}}$ & $14.5^{\mathrm{a}}$ & $18.5^{\mathrm{a}}$ & $16.0^{\mathrm{a}}$ & $1.5^{\mathrm{a}}$ & $1.5^{\mathrm{a}}$ & $1.3^{\mathrm{a}}$ & $1.1^{\mathrm{a}}$ \\
\hline \multicolumn{2}{|l|}{ Mean } & 14.3 & 14.8. & 16.1 & 15.8 & 1.4 & 1.5 & 1.2 & 1.2 \\
\hline \multirow[t]{5}{*}{ LSD } & & 1.5 & 2.5 & 0.9 & 1.4 & 0.1 & 0.4 & 0.1 & 0.1 \\
\hline & $\mathrm{CxG}$ & & & & & & & & \\
\hline & $\left.\mathrm{x} \mathrm{GA}_{3}(1)\right)$ & $*$ & $*$ & $*$ & $*$ & * & $*$ & $*$ & $*$ \\
\hline & $x \mathrm{GA}_{3}(2)$ & * & * & $*$ & $*$ & * & $*$ & $*$ & $*$ \\
\hline & $\left.\mathrm{x} \mathrm{GA}_{3}(0)\right)$ & $*$ & $*$ & $*$ & $*$ & $*$ & $*$ & $*$ & $*$ \\
\hline
\end{tabular}

Mean values for each cultivar separately in the same column followed by different letters differ significantly at $\mathrm{P}=0.05$.

*: statistically significant $(\mathrm{p}<0.05)$; ns: not significant.

$\mathrm{GA}_{3}(1)=50 \mathrm{mg} \mathrm{L}{ }^{-1}$ Gibberellic acid; $\mathrm{GA}_{3}(2)=100 \mathrm{mg} \mathrm{L}^{-1}$ Gibberellic acid; $\mathrm{GA}_{3}(0)=0 \mathrm{mg} \mathrm{L}-1$ Gibberellic acid.

\subsection{Effect of Gibberellic Acid on Plant Height}

$\mathrm{GA}_{3}$ application increased the mean plant height of all cultivars tested in the present study, although in cv. 'Pylaias' the increase observed was not statistically significant (Table 3). Plant height was significantly lower in 'Clemson' than in the other three cultivars. In both 'Clemson' and 'Boyiatiou' plant height was significantly increased by $\mathrm{GA}_{3}$ at both concentrations, whereas in 'Veloudo' the increase was only significant at the higher $\mathrm{GA}_{3}$ level $\left(\mathrm{GA}_{3}(2)\right)$. 
Table 3. The effect of gibberellic acid $\left(\mathrm{GA}_{3}\right)$ on mean plant height

\begin{tabular}{|c|c|c|}
\hline Cultivar (C) & $\begin{array}{l}\text { Gibberellic acid rate } \\
\quad\left(\mathrm{mg} \mathrm{L}^{-1}\right)\left(\mathrm{GA}_{3}\right)\end{array}$ & $\begin{array}{l}\text { Plant height } \\
(\mathrm{cm})\end{array}$ \\
\hline & $\mathrm{GA}_{3}(1)$ & $230.0^{\mathrm{a}}$ \\
\hline \multirow[t]{2}{*}{ 'Boyiatiou' } & $\mathrm{GA}_{3}(2)$ & $240.0^{\mathrm{a}}$ \\
\hline & $\mathrm{GA}_{3}(0)$ & $153.0^{\mathrm{b}}$ \\
\hline Mean & & 207.0 \\
\hline \multirow[t]{2}{*}{ LSD } & & 65.5 \\
\hline & $\mathrm{GA}_{3}(1)$ & $219.0^{\mathrm{ab}}$ \\
\hline \multirow[t]{2}{*}{ 'Veloudo' } & $\mathrm{GA}_{3}(2)$ & $242.0^{\mathrm{a}}$ \\
\hline & $\mathrm{GA}_{3}(0)$ & $206.0^{\mathrm{b}}$ \\
\hline Mean & & 222.0 \\
\hline \multirow[t]{2}{*}{ LSD } & & 32.0 \\
\hline & $\mathrm{GA}_{3}(1)$ & $188.0^{\mathrm{a}}$ \\
\hline \multirow[t]{2}{*}{ 'Pylaias' } & $\mathrm{GA}_{3}(2)$ & $200.0^{\mathrm{a}}$ \\
\hline & $\mathrm{GA}_{3}(0)$ & $178.0^{\mathrm{a}}$ \\
\hline Mean & & 188.0 \\
\hline \multirow[t]{2}{*}{ LSD } & & 36.0 \\
\hline & $\mathrm{GA}_{3}(1)$ & $162.0^{\mathrm{a}}$ \\
\hline \multirow[t]{2}{*}{ 'Clemson' } & $\mathrm{GA}_{3}(2)$ & $180.0^{\mathrm{a}}$ \\
\hline & $\mathrm{GA}_{3}(0)$ & $74.0^{\mathrm{b}}$ \\
\hline \multicolumn{2}{|l|}{ Mean } & 138.0 \\
\hline \multicolumn{2}{|l|}{ LSD } & 42.8 \\
\hline \multicolumn{3}{|c|}{$\mathrm{C} \times \mathrm{G}$} \\
\hline \multicolumn{2}{|c|}{$\left(\mathrm{C} \times \mathrm{GA}_{3}(1)\right)$} & $*$ \\
\hline \multicolumn{2}{|c|}{$\left(\mathrm{C} \times \mathrm{GA}_{3}(2)\right.$} & $*$ \\
\hline \multicolumn{2}{|c|}{$\left(\mathrm{Cx} \mathrm{GA}_{3}(0)\right)$} & $*$ \\
\hline
\end{tabular}

Mean values for each cultivar separately in the same column followed by different letters differ significantly at $\mathrm{P}=0.05$.

*: statistically significant $(\mathrm{p}<0.05)$; ns: not significant.

$\mathrm{GA}_{3}(1)=50 \mathrm{mg} \mathrm{L}{ }^{-1}$ Gibberellic acid; $\mathrm{GA}_{3}(2)=100 \mathrm{mg} \mathrm{L}^{-1}$ Gibberellic acid; $\mathrm{GA}_{3}(0)=0 \mathrm{mg} \mathrm{L}-1$ Gibberellic acid.

\subsection{Effect of Gibberellic Acid on the Number of Seeds Per Pod the 100 Seed Weight}

In all cultivars, the application of $\mathrm{GA}_{3}$ significantly increased the number of seeds per pod, irrespective of the time of harvest (Table 4). $\mathrm{GA}_{3}$ was equally effective at both concentrations used in this study In 'Boyiatiou' and 'Veloudo' the number of seeds per pod also tended to increase with increasing pod age at harvest.

The 100 seed weight of 'Pylaias' 'Clemson' and 'Veloudo' was not affected by $\mathrm{GA}_{3}$ application, irrespective of the time of harvest (Table 4). In 'Boyiatiou', there was no effect of $\mathrm{GA}_{3}$ on the mean 100 seed weight of seeds harvested $30 \mathrm{DAA}$, but thereafter the seeds from the control $\mathrm{GA}_{3}(0)$ had a higher 100 seed weight than those of seeds from the $\mathrm{GA}_{3}$ treatments, especially that with the high $\mathrm{GA}_{3}$ level $\left(\mathrm{GA}_{3}(2)\right)$. It is likely that the decrease in seed size (100 seed weight) correlated with the mean seed number, i.e. as seed number increased, mean 100 seed weight decreased. 
Table 4. The effect of gibberellic acid on the number of seeds per pod and the 100 seed weight in relation to harvesting time

\begin{tabular}{|c|c|c|c|c|c|c|c|c|c|}
\hline \multirow{2}{*}{$\begin{array}{l}\text { Cultivar } \\
\text { (C) }\end{array}$} & \multirow{2}{*}{$\begin{array}{l}\text { Gibberellic acid rate } \\
\left(\mathrm{mg} \mathrm{L}^{-1}\right)\left(\mathrm{GA}_{3}\right)\end{array}$} & \multicolumn{4}{|c|}{ Mean number of seeds/pod } & \multicolumn{4}{|c|}{ Mean 100 seed weight $(\mathrm{g})$} \\
\hline & & $\begin{array}{c}30 \\
\text { DAA }\end{array}$ & $\begin{array}{c}35 \\
\text { DAA }\end{array}$ & $\begin{array}{c}40 \\
\text { DAA }\end{array}$ & $\begin{array}{c}50 \\
\text { DAA }\end{array}$ & $\begin{array}{c}30 \\
\text { DAA }\end{array}$ & $\begin{array}{c}35 \\
\text { DAA }\end{array}$ & $\begin{array}{c}40 \\
\text { DAA }\end{array}$ & $\begin{array}{c}50 \\
\text { DAA }\end{array}$ \\
\hline & $\mathrm{GA}_{3}(1)$ & $42.9^{\mathrm{a}}$ & $58.0^{\mathrm{a}}$ & $55.7^{\mathrm{a}}$ & $56.1^{\mathrm{a}}$ & $21.3^{\mathrm{a}}$ & $26.3^{\mathrm{a}}$ & $32.0^{c}$ & $19.2^{\mathrm{b}}$ \\
\hline \multirow[t]{2}{*}{ 'Boyiatiou' } & $\mathrm{GA}_{3}(2)$ & $51.8^{\mathrm{a}}$ & $52.8^{\mathrm{a}}$ & $58.8^{\mathrm{a}}$ & $60.7^{\mathrm{a}}$ & $19.5^{\mathrm{a}}$ & $17.7^{\mathrm{b}}$ & $34.1^{\mathrm{b}}$ & $19.6^{\mathrm{b}}$ \\
\hline & $\mathrm{GA}_{3}(0)$ & $25.1^{\mathrm{b}}$ & $24.7^{b}$ & $31.8^{\mathrm{b}}$ & $20.5^{\mathrm{b}}$ & $21.3^{\mathrm{a}}$ & $26.8^{\mathrm{a}}$ & $37.5^{\mathrm{a}}$ & $23.3^{\mathrm{a}}$ \\
\hline Mean & & 39.9 & 45.1 & 48.7 & 45.7 & 34.5 & 23.6 & 20.7 & 20.7 \\
\hline \multirow[t]{2}{*}{ LSD } & & 15.6 & 17.7 & 19.4 & 15.4 & 4.0 & 4.4 & 1.0 & 3.1 \\
\hline & $\mathrm{GA}_{3}(1)$ & $34.2^{\mathrm{a}}$ & $43.2^{\mathrm{a}}$ & $40.5^{\mathrm{a}}$ & $58.8^{\mathrm{a}}$ & $25.6^{\mathrm{a}}$ & $29.0^{\mathrm{a}}$ & $39.7^{\mathrm{a}}$ & $22.4^{\mathrm{a}}$ \\
\hline \multirow[t]{2}{*}{ ‘Veloudo' } & $\mathrm{GA}_{3}(2)$ & $46.2^{\mathrm{a}}$ & $45.3^{\mathrm{a}}$ & $54.4^{\mathrm{a}}$ & $51.8^{\mathrm{a}}$ & $24.9^{\mathrm{a}}$ & $22.7^{\mathrm{b}}$ & $41.8^{\mathrm{a}}$ & $23.4^{\mathrm{a}}$ \\
\hline & $\mathrm{GA}_{3}(0)$ & $15.7^{\mathrm{b}}$ & $19.3^{b}$ & $22.8^{\mathrm{b}}$ & $29.2^{\mathrm{b}}$ & $26.8^{\mathrm{a}}$ & $33.5^{\mathrm{a}}$ & $39.4^{\mathrm{a}}$ & $23.7^{\mathrm{a}}$ \\
\hline Mean & & 32.0 & 35.9 & 39.2 & 46.6 & 40.3 & 28.4 & 25.7 & 23.1 \\
\hline \multirow[t]{2}{*}{ LSD } & & 12.7 & 14.1 & 16.5 & 11.7 & 3.9 & 4.6 & 3.2 & 1.4 \\
\hline & $\mathrm{GA}_{3}(1)$ & $58.9^{\mathrm{a}}$ & $54.5^{\mathrm{a}}$ & $52.6^{\mathrm{a}}$ & $48.1^{\mathrm{a}}$ & $22.7^{\mathrm{a}}$ & $32.5^{\mathrm{a}}$ & $39.4^{\mathrm{a}}$ & $25.4^{\mathrm{a}}$ \\
\hline \multirow[t]{2}{*}{ 'Pylaias' } & $\mathrm{GA}_{3}(2)$ & $57.9^{\mathrm{a}}$ & $57.1^{\mathrm{a}}$ & $53.8^{\mathrm{a}}$ & $54.7^{\mathrm{a}}$ & $23.4^{\mathrm{a}}$ & $34.4^{\mathrm{a}}$ & $40.0^{\mathrm{a}}$ & $24.2^{\mathrm{a}}$ \\
\hline & $\mathrm{GA}_{3}(0)$ & $31.0^{\mathrm{b}}$ & $27.9^{b}$ & $29.6^{\mathrm{b}}$ & $25.5^{\mathrm{b}}$ & $21.5^{\mathrm{a}}$ & $33.2^{\mathrm{a}}$ & $38.5^{\mathrm{a}}$ & $25.9^{\mathrm{a}}$ \\
\hline Mean & & 49.2 & 46.5 & 45.3 & 42.7 & 39.3 & 33.3 & 22.5 & 25.1 \\
\hline \multirow[t]{2}{*}{ LSD } & & 13.0 & 12.6 & 11.5 & 10.5 & 3.4 & 9.2 & 4.6 & 2.1 \\
\hline & $\mathrm{GA}_{3}(1)$ & $72.4^{\mathrm{a}}$ & $54.1^{\mathrm{a}}$ & $51.7^{\mathrm{a}}$ & $64.0^{\mathrm{a}}$ & $21.9^{\mathrm{a}}$ & $26.2^{\mathrm{a}}$ & $38.6^{\mathrm{a}}$ & $23.5^{\mathrm{a}}$ \\
\hline \multirow[t]{2}{*}{ 'Clemson' } & $\mathrm{GA}_{3}(2)$ & $62.8^{\mathrm{a}}$ & $62.5^{\mathrm{a}}$ & $62.5^{\mathrm{a}}$ & $60.1^{\mathrm{a}}$ & $21.4^{\mathrm{a}}$ & $26.5^{\mathrm{a}}$ & $36.4^{\mathrm{ab}}$ & $23.1^{\mathrm{a}}$ \\
\hline & $\mathrm{GA}_{3}(0)$ & $28.6^{\mathrm{b}}$ & $28.8^{b}$ & $29.6^{\mathrm{b}}$ & $34.0^{\mathrm{b}}$ & $20.3^{\mathrm{a}}$ & $20.8^{\mathrm{b}}$ & $34.8^{\mathrm{b}}$ & $23.6^{\mathrm{a}}$ \\
\hline \multirow{2}{*}{$\begin{array}{l}\text { Mean } \\
\text { LSD }\end{array}$} & & 54.6 & 23.5 & 47.9 & 52.7 & 36.6 & 24.5 & 21.2 & 23.4 \\
\hline & & 13.7 & 11.5 & 12.3 & 14.2 & 3.1 & 3.2 & 3.4 & 5.9 \\
\hline \multicolumn{10}{|c|}{$\mathrm{CxG}$} \\
\hline & $\left(\mathrm{C} \mathrm{x} \mathrm{GA}_{3}(1)\right)$ & * & $*$ & $*$ & * & $*$ & $*$ & $*$ & $*$ \\
\hline & $\left(\mathrm{C} \times \mathrm{GA}_{3}(2)\right.$ & $\mathrm{ns}$ & $\mathrm{ns}$ & * & * & $*$ & $*$ & $*$ & $*$ \\
\hline & $\left(\mathrm{C} \mathrm{x} \mathrm{GA}_{3}(0)\right)$ & $*$ & ns & ns & $*$ & $*$ & $*$ & $*$ & ns \\
\hline
\end{tabular}

Mean values for each cultivar separately in the same column followed by different letters differ significantly at $\mathrm{P}=0.05$.

*: statistically significant $(\mathrm{p}<0.05)$; ns: not significant.

$\mathrm{GA}_{3}(1)=50 \mathrm{mg} \mathrm{L}^{-1}$ Gibberellic acid; $\mathrm{GA}_{3}(2)=100 \mathrm{mg} \mathrm{L}^{-1}$ Gibberellic acid; $\mathrm{GA}_{3}(0)=0 \mathrm{mg} \mathrm{L}-1$ Gibberellic acid.

\subsection{Effect of Gibberellic Acid on Seed Germination, Seed Moisture Content and the Incidence of Hard Seeds}

The moisture content of seeds harvested 30 DAA was very high in all cultivars (49-55\%), but progressively decreased as harvesting was delayed, so that at 50DDA moisture content was just 12-13\% (Table 5). There was no consistent effect of $\mathrm{GA}_{3}$ application on seed moisture content, although at 50 DAA the moisture content of seeds from the $\left(\mathrm{GA}_{3}(2)\right)$ treatment of 'Veloudo' and $\left(\mathrm{GA}_{3}(1)\right)$ and $\left(\mathrm{GA}_{3}(2)\right)$ treatments of 'Clemson' was significantly lower than that of the control $\left(\mathrm{GA}_{3}(0)\right)$.

In 'Boyiatiou' seed germination was highest for pods harvested 35 DAA and decreased significantly for pods harvested 50 DAA. In the other cultivars, however, seed germination was low for pods harvested at 30 DAA, but higher for pods harvested at later dates (35-50 DAA) (Table 5). In 'Boyiatiou' germination was significantly lower in seeds harvested from plants treated with the lower $\mathrm{GA}_{3}$ concentration $\left(\mathrm{GA}_{3}(1)\right)$. In contrast, in 'Veloudo' germination was significantly higher in both $\mathrm{GA}_{3}$ treatments compared with the control, irrespective of harvest 
time, whereas in 'Pylaias' and 'Clemson;' no effect of $\mathrm{GA}_{3}$ on seed germination was detected. The incidence of hard seeds was highest in 'Boyiatiou' and lowest in 'Clemson'. In 'Veloudo' there appeared to be a reduction in the percentage of hard seeds within the $\mathrm{GA}_{3}$ treatments, which may account for the positive effect of $\mathrm{GA}_{3}$ on germination in this cultivar (Table 5).

Table 5. The effect of gibberellic acid on seed moisture content at harvest, seed germination and seed hardness in relation to harvesting time

\begin{tabular}{|c|c|c|c|c|c|c|c|c|c|c|}
\hline \multirow{3}{*}{$\begin{array}{l}\text { Cultivar } \\
\text { (C) }\end{array}$} & \multirow{3}{*}{$\begin{array}{l}\text { Gibberellic acid rate } \\
\left(\mathrm{mg} \mathrm{L}^{-1}\right)\left(\mathrm{GA}_{3}\right)\end{array}$} & \multicolumn{4}{|c|}{$\begin{array}{c}\text { Mean moisture content at harvest } \\
(\%)\end{array}$} & \multicolumn{4}{|c|}{$\begin{array}{l}\text { Mean germination } \\
(\%)\end{array}$} & \multirow{3}{*}{$\begin{array}{c}\text { Seed hardnes } \\
(\%)\end{array}$} \\
\hline & & 30 & 35 & 40 & 50 & 30 & 35 & 40 & 50 & \\
\hline & & DAA & DAA & DAA & DAA & DAA & DAA & DAA & DAA & \\
\hline & $\mathrm{GA}_{3}(1)$ & $48.1^{\mathrm{b}}$ & $34.7^{\mathrm{a}}$ & $14.5^{\mathrm{a}}$ & $13.5^{\mathrm{a}}$ & $26.4^{\mathrm{b}}$ & $48.9^{\mathrm{b}}$ & $40.5^{b}$ & $23.0^{\mathrm{b}}$ & 54.8 \\
\hline \multirow[t]{2}{*}{ 'Boyiatiou' } & $\mathrm{GA}_{3}(2)$ & $52.8^{\mathrm{a}}$ & $20.3^{\mathrm{b}}$ & $12.4^{\mathrm{a}}$ & $13.9^{\mathrm{a}}$ & $38.8^{\mathrm{a}}$ & $69.0^{\mathrm{a}}$ & $64.2 \mathrm{a}$ & $31.3^{\mathrm{a}}$ & 48.7 \\
\hline & $\mathrm{GA}_{3}(0)$ & $48.7^{\mathrm{b}}$ & $30.0^{\mathrm{a}}$ & $15.2^{\mathrm{a}}$ & $12.9^{\mathrm{a}}$ & $40.5^{\mathrm{a}}$ & $70.5^{\mathrm{a}}$ & $61.6^{\mathrm{a}}$ & $33.1^{\mathrm{a}}$ & 45.0 \\
\hline Mean & & 49.8 & 28.3 & 14.0 & 13.4 & 35.2 & 62.8 & 55.4 & 29.1 & 49.5 \\
\hline \multirow[t]{2}{*}{ LSD } & & 4.2 & 8.7 & 4.2 & 2.1 & 5.9 & 6.2 & 6.9 & 5.2 & \\
\hline & $\mathrm{GA}_{3}(1)$ & $51.8^{\mathrm{c}}$ & $35.6^{\mathrm{a}}$ & $19.4^{\mathrm{a}}$ & $12.6^{\mathrm{a}}$ & $34.8^{\mathrm{a}}$ & $72.6^{\mathrm{a}}$ & $72.1^{\mathrm{a}}$ & $72.1^{\mathrm{a}}$ & 20.0 \\
\hline \multirow[t]{2}{*}{ 'Veloudo' } & $\mathrm{GA}_{3}(2)$ & $55.8^{\mathrm{a}}$ & $25.3^{\mathrm{b}}$ & $18.4^{\mathrm{a}}$ & $10.6^{\mathrm{b}}$ & $35.7^{\mathrm{a}}$ & $70.6^{\mathrm{a}}$ & $72.2^{\mathrm{a}}$ & $74.3^{\mathrm{a}}$ & 17.7 \\
\hline & $\mathrm{GA}_{3}(0)$ & $53.8^{\mathrm{b}}$ & $41.6^{\mathrm{a}}$ & $22.2^{\mathrm{a}}$ & $12.9 \mathrm{a}$ & $23.5^{\mathrm{b}}$ & $46.6^{\mathrm{b}}$ & $52.2^{\mathrm{b}}$ & $44.9^{\mathrm{b}}$ & 32.8 \\
\hline Mean & & 53.8 & 34.1 & 20.0 & 12.0 & 31.3 & 63.2 & 65.5 & 63.7 & 23.5 \\
\hline \multirow[t]{2}{*}{ LSD } & & 1.6 & 10.0 & 8.7 & 1.8 & 4.9 & 5.4 & 5.9 & 6.3 & \\
\hline & $\mathrm{GA}_{3}(1)$ & $53.5^{\mathrm{a}}$ & $38.4^{\mathrm{a}}$ & $18.4^{\mathrm{a}}$ & $11.7^{\mathrm{a}}$ & $38.7^{\mathrm{a}}$ & $84.1^{\mathrm{a}}$ & $86.1^{\mathrm{a}}$ & $85.4^{\mathrm{a}}$ & 10.6 \\
\hline \multirow[t]{2}{*}{ 'Pylaias' } & $\mathrm{GA}_{3}(2)$ & $53.8^{\mathrm{a}}$ & $41.8^{\mathrm{a}}$ & $17.2^{\mathrm{a}}$ & $12.2^{\mathrm{a}}$ & $39.6^{\mathrm{a}}$ & $84.0^{\mathrm{a}}$ & $87.7^{\mathrm{a}}$ & $83.6^{\mathrm{a}}$ & 12.0 \\
\hline & $\mathrm{GA}_{3}(0)$ & $54.3^{\mathrm{a}}$ & $44.1^{\mathrm{a}}$ & $17.8^{\mathrm{a}}$ & $12.9^{\mathrm{a}}$ & $43.5^{\mathrm{a}}$ & $88.3^{\mathrm{a}}$ & $86.1^{\mathrm{a}}$ & $80.6^{\mathrm{a}}$ & 14.0 \\
\hline Mean & & 53.8 & 41.4 & 17.8 & 12.2 & 40.6 & 85.4 & 86.6 & 83.2 & 12.2 \\
\hline \multirow[t]{2}{*}{ LSD } & & 2.6 & 6.3 & 8.5 & 1.3 & 7.0 & 5.7 & 3.7 & 5.2 & \\
\hline & $\mathrm{GA}_{3}(1)$ & $55.1^{\mathrm{a}}$ & $28.6^{\mathrm{a}}$ & $12.9^{\mathrm{b}}$ & $11.9^{\mathrm{b}}$ & $37.5^{\mathrm{b}}$ & $77.3^{\mathrm{a}}$ & $91.0^{\mathrm{b}}$ & $88.7^{\mathrm{a}}$ & 5.3 \\
\hline \multirow[t]{2}{*}{ 'Clemson' } & $\mathrm{GA}_{3}(2)$ & $55.1^{\mathrm{a}}$ & $28.5^{\mathrm{a}}$ & $16.9^{\mathrm{a}}$ & $11.6^{\mathrm{b}}$ & $38.8^{\mathrm{b}}$ & $75.9^{\mathrm{a}}$ & $93.8^{\mathrm{a}}$ & $91.3^{\mathrm{a}}$ & 4.3 \\
\hline & $\mathrm{GA}_{3}(0)$ & $54.0^{\mathrm{a}}$ & $33.0^{\mathrm{a}}$ & $12.8^{\mathrm{b}}$ & $13.1^{\mathrm{a}}$ & $60.9^{\mathrm{a}}$ & $77.9^{\mathrm{a}}$ & $94.9^{\mathrm{a}}$ & $91.3^{\mathrm{a}}$ & 4.8 \\
\hline Mean & & 54.7 & 30.0 & 14.2 & 12.2 & 45.7 & 77.0 & 93.2 & 90.4 & 4.8 \\
\hline \multirow[t]{5}{*}{ LSD } & & 2.7 & 13.7 & 3.6 & 0.9 & 6.4 & 2.7 & 2.7 & 3.8 & \\
\hline & $\mathrm{Cx} \mathrm{G}$ & & & & & & & & & \\
\hline & $\left(\mathrm{Cx} \mathrm{GA}_{3}(1)\right)$ & $*$ & $*$ & $*$ & $*$ & $*$ & $*$ & $*$ & $*$ & \\
\hline & $\left(\mathrm{C} \times \mathrm{GA}_{3}(2)\right.$ & $\mathrm{ns}$ & * & * & $*$ & * & $*$ & * & * & \\
\hline & $\left(\mathrm{Cx} \mathrm{GA}_{3}(0)\right)$ & * & $*$ & $*$ & $*$ & $*$ & $*$ & $*$ & $*$ & \\
\hline
\end{tabular}

Mean values for each cultivar separately in the same column followed by different letters differ significantly at $\mathrm{P}=0.05$.

*: statistically significant $(\mathrm{p}<0.05)$; ns: not significant.

$\mathrm{GA}_{3}(1)=50 \mathrm{mg} \mathrm{L}-1$ Gibberellic acid; $\mathrm{GA}_{3}(2)=100 \mathrm{mg} \mathrm{L}-1$ Gibberellic acid; $\mathrm{GA}_{3}(0)=0 \mathrm{mg} \mathrm{L}{ }^{-1}$ Gibberellic acid.

\subsection{Effect of Storage On Seed Germination}

The storage of seeds for 18 months remarkably improved the germination of all cultivars, but especially 'Veloudo' and 'Boyiatiou' where the increase in germination after storage was was statistically significant (increased by $41 \%$ and $33 \%$ respectively) (Figure 2). 


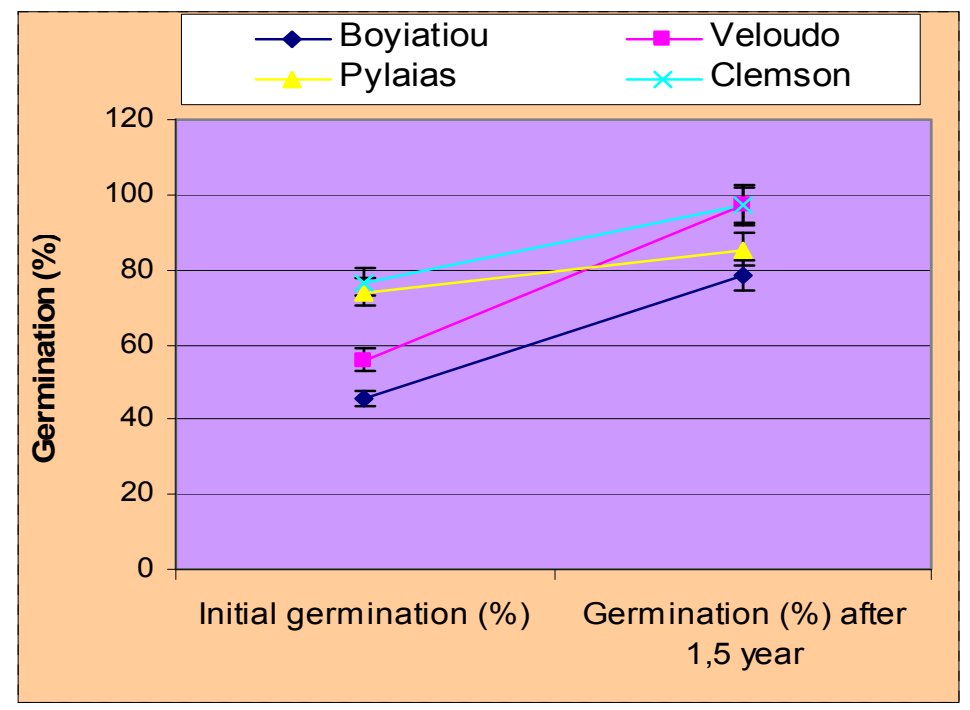

Figure 2. Seed germination percentage at harvest time and after 18 months storage at room temperature of $25{ }^{\circ} \mathrm{C}$ (pooled data for all treatments)

\section{Discussion}

Although $\mathrm{GA}_{3}$ application at an early stage in okra growth resulted in a significant increase in plant height (Table 3 ), it did not cause a parallel increase in flower induction or pod set (Table 1). Similarly, $\mathrm{GA}_{3}$ did not significantly affect pod dimensions (length and diameter) (Table 2), which were primarily determined by the genotype (cultivar), but it did significantly increase the number of seeds per pod, all at the expense of seed size in 'Boyiatiou' (Table 4).

The positive effect of gibberellin on plant height is well documented for okra and for other species. In okra, $\mathrm{GA}_{3}$ increases plant height by increasing intermodal length, but has no effect on the number of nodes per plant. Since flowers are induced singly at each node (Dhingra, 2009), there is therefore no effect of $\mathrm{GA}_{3}$ on flower or pod induction. Similarly, the application of Gibberellin inbitors, e.g. chlormequat chloride, reduce internode length and therefore plant height, but with no positive effect on node or flower induction (Thanopoulos, Petropoulos, Alexopoulos, Karapanos, Khah, Akoumianakis \& Passam, 2013). The effect of $\mathrm{GA}_{3}$ may be exerted from an early stage in plant growth, such as the seedling (Pal and Hossain, 2001) or even the seed (Das \& Pattanaik, 1971). In other studies, $\mathrm{GA}_{3}$ has been found to have no effect on pod induction (Syed, Hussain, Nawab, Asghar \& Ali, 1997), although an increase in flowering, but not the percentage of fruit set, was observed when $\mathrm{GA}_{3}$ was applied as a seed soak (Nandpuri et al., 1969). In contrast, Abduljabar et al. (2007) reported an increase in okra seed production when the plant apex was removed and $\mathrm{GA}_{3}(100 \mathrm{ppm})$ applied one day later. The increase in seed yield was apparently a result of $\mathrm{GA}_{3}$-promoted side shoot development and is therefore not comparable with the present experiment where the main shoot was allowed to grow continually.

The lack of effect of $\mathrm{GA}_{3}$ on pod growth (length and diameter) is not surprising since this characteristic is influenced mainly by genotype (Raji, 1994). A decrease in pod size when harvesting is delayed (50 DAA) may be attributed to pod desiccation under the conditions of high temperatures and low humidity experienced in the field (Figure 1). Kokare, Bhalerao, Prabu, Chavan, Bansode and Kachare (2006) and Pal and Hossain (2001) also noted an absence of effect of plant growth regulators on okra pod growth, although Asghar et al. (1997) and Dhankhar and Singh (2009) suggested an increase in pod length and diameter following $\mathrm{GA}_{3}$ application. In these studies, however, pods were harvested at a smaller size, i.e. before full maturation, as here. Moroeover, the effect of $\mathrm{GA}_{3}$ may depend on the timing and number of applications made, as shown by Ayyub, Ahmad and Akhtar (2013) who reported an increase in fresh pod yield when okra was repeatedly sprayed with GA throughout the growing season.

The principal effect of $\mathrm{GA}_{3}$ on okra in the present experiment was the significant increase in the number of seeds per pod (Table 5). However, Bhatt and Srinivasa (1998) found no effect of $\mathrm{GA}_{3}$ on the seed number per pod in okra, possibly due to the fact that they used a lower concentration of $\mathrm{GA}_{3}\left(5050\right.$ and $\left.100 \mathrm{mg} \mathrm{L}^{-1}\right)$ compared with that used here. Although an increase in the number of seeds per pod is of immediate concern for seed producers, it may nevertheless result in lower seed quality, as indicated by the significantly lower 100 seed weights of seeds of 'Boyiatiou' from the $\mathrm{GA}_{3}$ treated plants (Table 5). Mean 100 seed weight at harvest depends mainly on the cultivar 
and on the extent of seed drying. In the present experiment seeds harvested 50 DAA had a significantly lower mean 100 seed weight than those harvested earlier (especially at 30 DAA). Although Singh et al. (1999) indicated an increase in okra seed weight per pod following foliar application of $\mathrm{GA}_{3}(20-40 \mathrm{ppm})$, this result was obtained when seeds were produced on plants that had already been harvested for fresh pods.

In general, there was little or no effect of $\mathrm{GA}_{3}$ on seed moisture content at harvest. Seeds rapidly dried as pod harvesting was delayed, especially at 40-50 DAA. A negative effect of rapid drying under high field temperatures is an increase in the percentage of hard seeds (Demir, 1997). This is particularly the case in cv. 'Boyiatiou' and to some extent 'Veloudo'. In 'Veloudo' there was some indication that $\mathrm{GA}_{3}$ may reduce the incidence of hard seeds, thereby improving seed quality, as shown by the higher germination of seeds from $\mathrm{GA}_{3}$ treated plants in this cultivar (Table 5). A similar result has been reported for cv. other cultivars (Patil, Kadam, \& Kolase, 2007) and cv. 'Balady' grown under winter conditions in Egypt. There was, however, no effect of $\mathrm{GA}_{3}$ on seed germination (i.e. seed quality) in either 'Clemson' or 'Pylaias', whereas in 'Boyiatiou' germination was significantly lower in seeds harvested from plants treated with the lower $\mathrm{GA}_{3}$ concentration $\left(\mathrm{GA}_{3}(1)\right)$. The reason for this effect of $\mathrm{GA}_{3}$ is not clear since it occurred only at the lower $\mathrm{GA}_{3}$ level $\left(50 \mathrm{mg} \mathrm{L}^{-1}\right)$ and not the higher level $\left(100 \mathrm{mg} \mathrm{L}^{-1}\right)$; it cannot therefore be a toxicity effect. It did, however, occurs at all stages of harvest and therefore requires further investigation.

It should be noted that because $\mathrm{GA}_{3}$ was applied only once to plants at an early stage of growth, the results described here refer to pods formed on the lower part of the plant, where $\mathrm{GA}_{3}$ would be expected to have maximum effect. It is known that the sensitivity of plants to $\mathrm{GA}_{3}$ varies with the stage of growth at the time of application (Moore, 1979). Consequently, $\mathrm{GA}_{3}$ applied to okra at the 3-4 leaf stage might be expected to affect pods and seeds formed early on in the life cycle of the plant (i.e. in the lower part of the mature plant) but not necessarily later on. When seed germination was recorded in pods from different positions on the plant (data not presented), it was observed that in all cultivars except 'Clemson' germination was higher in seeds from pods harvested from the lower part of the plant than those from pods in the upper part of the plant, irrespective of $\mathrm{GA}_{3}$ treatment. Additionally, the percentage of hard seeds in all cultivars except 'Clemson' was higher in seeds from the upper part of the plant, clearly indicating that the reduction of germination observed here was due to an increase in seed hardness. We cannot, however, attribute these differences to $\mathrm{GA}_{3}$ application because the pods from the lower and upper parts of the plant were harvested at different times and therefore subjected to different climatic conditions. Rather, it appears that pods and seeds from the upper plant part matured under higher temperatures and lower humidity; in consequence seeds dried more rapidly than those form the lower part of the plant, leading to the formation of a hard seed testa (Demir, 1997). These considerations were not necessarily taken into account in other experiments where $\mathrm{GA}_{3}$ was applied to the seed or seedling and measurements taken at the end of the biological cycle of the crop (Das \& Pattanaik, 1971; Singh et al., 1999).

\section{Conclusion}

Application of $\mathrm{GA}_{3}$ at a concentration of $50-100 \mathrm{mg} \mathrm{L}^{-1}$ to okra at the 3-4 leaf stage increased plant height irrespective of cultivar, but without increasing flower induction or pod set. Similarly, $\mathrm{GA}_{3}$ had no effect on pod dimensions (length, diameter) or mean 100 seed weight, except in Boyiatiou. Similarly, $\mathrm{GA}_{3}$ application did not consistently affect seed moisture content, but it did however, increase the number of seeds per pod. Germination was either promoted ('Veloudo'), inhibited ('Boyiatiou') or not affected ('Pylaias', 'Clemson') by GA Differences in germination were apparently related to the incidence of hard seeds. Storage of seeds for 18 months improved germination. It therefore seems that foliar application of $\mathrm{GA}_{3}$ may be beneficial for seed production of 'Veloudo' by increasing seed quality (germination), but is not of value for the other cultivars.

\section{References}

Abduljabbar, I. M., Abduljabbar, I., \& Shukri, H. S. (2007). Effect of sowing date, topping and some growth regulators on growth, pod and seeds yield of okra (Abelmoschus esculentus L.M.). African Crop Science Conference Proceedings, 8, 473-478.

Asghar, S., Hussain, S. A., \& Ali, N. (1997). Effect of exogenous growth regulators on growth, flowering and yield of okra (Abelmoschus esculentus L.). Sarhad Journal of Horticulture, 13(5), 449-453.

Ayyub, C. M., Ahmad, S., \& Akhtar, N. (2013). The effect of pre sowing magnetic treatment of okra seed on growth. Germany: Lambert Academic Publishing.

Bewley, J. D., \& Black, M. (1983). Physiology and biochemistry of seeds in relation to germination. New York: Springer-Verlag. 
Bhatt, R. M., \& Srinivasa, R. N. K. (1998). Germination response to fruit position and temperature in okra Abelmoschus esculenthus (L.). Indian Journal of Horticulture, 55, 81-84.

Briant, R. E. (1974). An analysis of the effects of gibberellic acid on tomato leaf growth. Journal of Experimental Botany, 25, 764-771. http://dx.doi.org/10.1093/jxb/25.4.764

Das, R. C., \& Pattanaik, A. (1971). Studies on the effect of growth regulator treated okra seeds Abelmoschus esculentus (L.) M. with respect to growth and subsequent development. Indian Journal of Horticulture, 28(4), 293-295.

Demir, I. (1997). Occurrence of hardseededness in relation to seed development in okra (Abelmoschus esculentus (L) Moench). Plant Varieties and Seeds, 10(1), 7-13.

Dhankhar, B. S., \& Singh, R. (2009). Okra hand book, global production, processing and crop improvement (p. 475). HNB Publishing.

Dhingra, R. H. (2009). Morphological features and reproductive biology. In B. S. Dhankhar \& R. Singh (Eds.), Okra handbook: Global production, processing and crop improvement (pp. 25-35). HNB Publishing. New York, USA.

Dijkstra, P., \& Kuiper, P. J. C. (1989). Effects of exogenously applied growth regulators on shoot growth of inbred lines of Plantago major differing in relative growth rate: Differential response to gibberellic acid and (2-chloroethyl) -trimethyl-ammonium chloride. Physiologia Plantarum, 77, 512-518. http://dx.doi.org/ 10.1111/j.1399-3054.1989.tb05385.x

Ilias, I., Ouzounidou, G., Giannakoula, A., \& Papadopoulou, P. (2007). Effects of gibberellic acid and prohexadione-calcium on growth, chlorophyll fluorescence and quality of okra plant. Biologia Plantarum, 51(3), 575-578. http://dx.doi.org/10.1007/s10535-007-0126-5.

ISTA. (2009). International rules for seed testing. Seed Sci. \& Technol. International Seed Testing Association Switzerland.

Kokare, R. T., Bhalerao, R. K., Prabu, T., Chavan, S. K., Bansode, B., \& Kachare, G. S. (2006). Effect of plant growth regulators on growth, yield and quality of okra (Abelmoschus esculentus (L.) Moench). Agricultural Science Digest, 26(3), 178-181.

Manzurul, M. H., \& Shahidul, M. H. (2002). Effects of gibberellic acid (GA3) on physiological contributing characters of mungbean (Vigna radiata L.). Pakistan Journal of Biological Sciences, 5(4), 401-403. http://dx.doi.org/10.3923/pjbs.2002.401.403.

Mohammadi, G., Khah, E. M., \& Bannayan, A. M. (2011). Differential responses for harvesting times and storage on hardness of different varieties of okra. Notulae Scientia Biologicae, 3(4), 117-122.

Moore, T. C. (1979). Biochemistry and physiology of plant hormones (pp. 48-50). New York: Springer-Verlag. http://dx.doi.org/10.1007/978-1-4684-0079-3

Nandpuri, K. S., Sooch, B. S., \& Randhawa, K. S. (1969). Effect of gamma irradiation on storage life and quality of onion bulbs under ordinary storage conditions. Journal of Research Punjab Agricultural University, 6, 755-762.

Pal, A. K, \& Hossain, M. (2001). Effect of seed soaking on growth, pod yield and seed yield in okra (Abelmoschus esculentus (L.) Moench.). Horticultural Journal, 14, 61-65.

Passam, H. C., \& Polyzou, P. (1997). Improvement of okra seed germination by acid, osmoconditioning and hot water treatments. Plant Varieties and Seeds, 10, 135-140.

Patil, R. V., Kadam, K. G., \& Kolase, S. K. (2007). Effect of plant growth regulators on seed yield and quality of okra (Abelmoschus esculentus (L.) Moench.). Ecology, Environment and Conservation, 13(4), 841-842.

Raji, J. A. (1994). Field and glass-house screening of okra genotypes for yield and intercropping. Indian Journal of Agricultural Sciences, 64(7), 454-458.

Syed, A., Hussain, S. A., Nawab, A., Asghar, S., \& Ali, N. (1997). Effect of exogenous growth regulators on growth, flowering and yield of okra (Abelmoschus esculentus L.). Sarhad Journal of Agriculture, 13, 449-453.

Thanopoulos, C., Petropoulos, S. A., Alexopoulos, A. A., Karapanos, I. C., Khah, E. M., Akoumianakis, K. A., ...Passam, H. C. (2013). A comparison of the effectiveness of chlormequat chloride (CCC) application 
and terminal apex excision to restrict plant height in okra (Abelmoschus esculentus [L.] Moench.) and optimize yield. Journal of Agricultural Science, 5(9), 44-50.

Yuan, L., \& Xu, D. Q. (2001). Stimulation effect of gibberellic acid short term treatment on leaf photosynthesis related to the increase in Rubisco content in broad bean and soybean. Photosynthesis Research, 68, 39-47. http://dx.doi.org/10.1023/A:1011894912421

\section{Copyrights}

Copyright for this article is retained by the author(s), with first publication rights granted to the journal.

This is an open-access article distributed under the terms and conditions of the Creative Commons Attribution license (http://creativecommons.org/licenses/by/3.0/). 